\title{
Survey on Flooding Detection System using Internet of Things
}

\author{
Mahendra Salunke \\ Assistant Professor, Pimpri Chinchwad College of \\ Engineering and Research, Ravet, Pune, \\ Maharshatra, India
}

\author{
Nilesh Korade \\ Assistant Professor, Pimpri Chinchwad College of \\ Engineering and Research, Ravet, Pune, \\ Maharshatra, India
}

\begin{abstract}
To decrease the challenges that the cities face such as scarcity of energy sources, flooding prevention, healthcare, housing water and deteriorating infrastructure, making a city 'Smart' is emerging. The Internet of Things or IOT provides the ability for human and machines to interact from billions of things that include sensors, services or other Internet connected things. This paper aims to realize the security requirement and security architecture of Internet of things technology for urban flooding prevention management system and discussed the demand and overall design of urban flooding prevention management system. Finally, the application process of the Internet of things technology in Chongqing flooding prevention management system is summarized. For emergency command and dispatch there is visual management, and at the same time, network assessment management for the drainage pipe can be conducted correctly. The flood control and drainage function of Chongqing will gradually improve with smooth drainage facilities also the inspection and maintenance management will standardized.
\end{abstract}

\section{Keywords}

Flooding Prevention, Internet of Things, Drainage pipe, catastrophic situation, flooding condition, Flow Rate, Water Level

\section{INTRODUCTION}

The term IOT is the inter-networking of physical devices such as vehicles, buildings, or embedded items such as software, sensors, actuators, and to enable these objects to collect and exchange data network connectivity is needed. The concept Internet of Things describes a future where every day physical objects can be connected to the Internet and also is able to identify themselves to other devices. IoT is closely identified with sensor technologies, RFID, wireless technologies. Internet is a medium that connect people across the world for emailing, gaming, conferencing etc. Internet of Things allows sensing of objects and controlling objects remotely across existing network infrastructure. Floods, extreme weather events, have occurred with frequent regularity over last two decades causing severe urban flood related inundations. India is primarily an agricultural country and rural infrastructure was adequate to sustain country population. Expansion of urban sector increased due to migration of population towards mega cities. Such migrations are due to industrial growth. Every year floods affect nearly 400 million hectares of land in India. Now a day's weather information is used for monitoring and warning on urban flooding. The citizens get notification when of people check, people on vehicles check or camera check, which has the defects of poor continuity, little data, slow speed and time lag. The people living in apartment don't get waterlog information exactly and quickly and thus they are not able to take corresponding measures and make warnings [1][2]. In the present scenario, due to migration of people from rural, rapidly expanding mega cities are facing many problems. The four major cities Mumbai, Delhi, Kolkata and Chennai are the most populated cities of India. Vulnerability of flooding increased due to uncontrolled growth of mega cities. For a better understanding of the problem, extreme rainfall events have been analyzed in the four Mega Cities [2]. Urban flooding is a mechanism of water disaster or flooding in urban caused due to continuous rainfall which exceeds the urban drainage capacity. Most of the rain water rivers flowing on urban ground, due to increasing waterproof ground which caused serious urban flooding in many cities. The rainstorm also makes urban flooding more serious, which need urban flood prevention and control, causes property loss, personnel casualties restrict city's development and other disasters that affect citizen's work and life[3]. Many developed countries conducting researches on urban waterlog, using computer aided way to monitor, make analogue simulations and forewarn. They have made urban flooding monitoring and forewarning system based on their geographic information system and own environmental simulation model. This paper studies the use of Internet of things in management of urban flooding to save life of citizens. A summary of casualties due to floods /heavy rains is shown in Table [2].

Table1. Number of Deaths/Injured in four mega cities

\begin{tabular}{|c|c|c|c|c|}
\hline \multirow[t]{2}{*}{ Year } & \multicolumn{4}{|c|}{ Deaths } \\
\hline & Delhi & Kolkata & Mumbai & Chennai \\
\hline 1990 & 3 & 1 & 79 & 15 \\
\hline 1995 & 2 & 6 & 7 & 12 \\
\hline 2000 & - & - & 87 & - \\
\hline 2005 & - & 16 & 1000 & 13 \\
\hline
\end{tabular}

\section{LITERATURE SURVEY}

\subsection{Application of Internet of Things in Urban Flooding Prevention Management System}

The Survey has analyzed the security architecture and security requirement of IOT technology. The paper describes the demand of urban waterlogging prevention management system with the help of IOT. The basic model urban waterlogging prevention has described in this article [1].

\subsection{Urban flooding in recent decades in four mega cities of India}

People are migrating from rural to urban area due to 
unemployment and other reasons. The population count is increasing day by day and due to that cities are facing many new challenges. Flooding condition is one of the big challenges increased due to uncontrolled growth of mega cities. The article describe the population count and death rate due to flooding in four mega cities in India[2].

\section{ARCHITECTURE}

The flooding Prevention Management System work in three dimension, calculation of drainage flow, rainfall, and water level, second sending collected flooding information to data center for analysis and monitoring and alerting mechanism. Use the data reasonably on the management and control platform, while constructing the system and apply the data fusion technology and wireless remote intelligent monitoring technology well.

\subsection{System Function Design}

There are five layers for the system, as shown in Figure 1. Good design management of user layer and application layer should be made. To determine the system design of perception layer data layer and network layer shall also be conducted.

\begin{tabular}{ll|} 
User & Government or Public Department \\
Application & Drainage Management System \\
\cline { 2 - 2 } Data & Geographical and Emergency \\
Network & Internet Mobile Network \\
& \\
Perception & Video Monitoring and Sensors
\end{tabular}

Fig 1. Flooding Detection System Requirement

\section{Drainage management}

An import analysis is needed based on the design of systematic drainage management system. The flow-meter calculates the rate of flow of water; simultaneously Water Level Indicator calculates the rising water level and rain sensor calculate the rainfall rate. The collected data is send to data center for analysis to determine flood condition. The analysis can also used to maintenance of drainage pipe.

\section{Data Center}

The data center performs statistical analysis on data collected from various sources. The analysis used for evaluation of flood condition and by Early Warning Subsystem.

\section{Emergency command and dispatch}

It is mainly designed in combination with the online flood situation; conduct well the analysis and management of operational data. The system should provide feedback and release flood information timely, and analyze the operation and dispatching situation of drainage facilities.

\subsection{Components}

\section{Ultrasonic Flow-meter}

An ultrasonic flow-meter is a volumetric flow meter which requires bubbles in the flow. Ultrasonic flow-meters are ideal for wastewater applications or any dirty liquid which is conductive or water based. It is a type of flow meter that measures the velocity of a fluid with ultrasound to calculate volume flow.

\section{Water Level Indicator}

Water Level Indicator detects the water level from ground or surface to determine flood condition.

\section{Rain sensor}

A rain sensor is a switching device that gets activated by rainfall. The rain sensor detects water that completes the circuits on its sensor boards' printed leads. The sensor board acts as a variable resistor that will change from 100k ohms when wet to $2 \mathrm{M}$ ohms when dry. In short, the wetter the board, more current will be conducted. The rain sensor detects the velocity of rainfall.

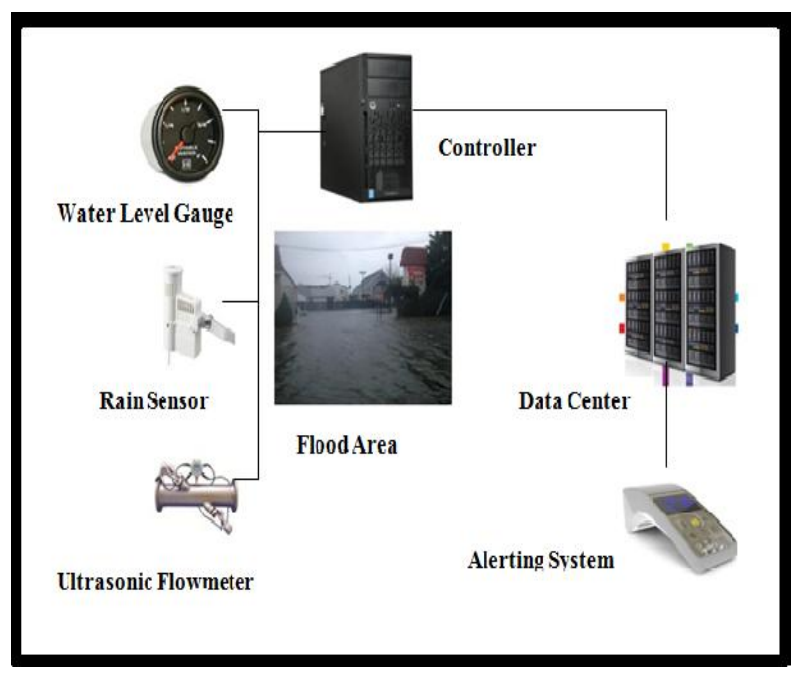

Fig 2. Flooding Detection System Using Internet of Things

\section{MATHEMATICS}

$\mathrm{f}=\{\mathrm{f} 1, \mathrm{f} 2, \mathrm{f} 3 \ldots\}$ set of water flow rate measured by ultrasonic flow meter after fixed interval.

$l=\{11,12,13\}$ set of water level measured by Water Level Indicator after fixed interval.

$\mathrm{r}=\{\mathrm{r} 1, \mathrm{r} 2, \mathrm{r} 3\}$ set of rain fall rate measured by Rain sensor after fixed interval.

$\mathrm{t}=$ Threshold value for catastrophic situation.

$\mathrm{r} \propto \mathrm{f}$ if rate of rainfall increase the water flow rate also increase due to pressure created by water level on drainage system .

$1 \propto \mathrm{f}$ if water level increase the water flow rate also increase. If water flow level cross a threshold and rain fall rate is also increasing or constant then there may be catastrophic situation.

if $\mathrm{f} / \mathrm{l}=\mathrm{constant}$ then there is less chances of catastrophic situation or flooding condition.

if $\mathrm{f} / \mathrm{l}>=\mathrm{t}$ then there may be catastrophic situation or flooding condition.

F- Water flow rate

R- Rainfall Rate

A-Area

T-Fixed Time Interval 


\section{P- Perimeter of Drainage Pipe}

Collected Water $=\mathrm{R} * \mathrm{~A} * \mathrm{~T}$

Water Dispatched $=\mathrm{F}^{*} \mathrm{P} * \mathrm{~T}$

Remaining Water to Dispatch $(\mathrm{RWD})=$ Collected WaterWater Dispatched

$\mathrm{RWD}=\sum_{\mathrm{t}=\mathrm{o}}^{\mathrm{n}} \mathrm{Rt} * A t^{*} \mathrm{~T}-\sum_{\mathrm{t}=\mathrm{o}}^{\mathrm{n}} \mathrm{F}_{\mathrm{t}} * \mathrm{P} * \mathrm{~T}$

Water Level $=$ RWD $/ \mathrm{A}$

\subsection{Algorithm}

step 1 : if rainfall then

step 2 : Activate the system, initialize WaterLevel, FlowRate, RainFall with its default values.

step 3 : do repeatedly

step 4 : calculate NewWaterLevel, NewFlowRate, NewRainFallRate.

step 5 : if NewRainFallRate > RainFallRate Then

step $6:$ if NewFlowRate $=$ FlowRate AND NewWaterLevel $>$ WaterLevel Then

step 7 : check threshold and do the necessary action for catastrophic situation or flooding condition.

step 8 : end if

step 9 : if NewFlowRate> FlowRate OR NewWaterLevel> WaterLevel OR NewFlowRate> FlowRate Then

step 10 : calculate probability of catastrophic situation by doing statistical analysis on available data.

step 11 : end if

step 12 : if NewFlowRate $=$ FlowRate then

step 13 : drainage capacity gets full or there is obstacle in drainage system, check RainFallRate to determine flooding condition.

step 14 : end if

step $15:$ if RainFallRate $==0$

step 16 : stop system

step 17 : end if

step 18 : continue with step 3 .

step 19 : end if

step 20 : end if

The FlowRate vary because of two reason, it's gets decrease when there is overflow in river or sea or if there is any obstacle in flow i.e. Chongqing flood control tunnel, and increase as level of water rises.

\section{APPLICATIONS}

The system can be used in high density area where ground floor are mostly used for parking cars and bikes. The system will alert to car owners before ground level gets filled by rain water and they can do alternative arrangement.. In Jammu \&
Kashmir, there are longer tunnels that are under construction, Panjal Railway Tunnel it is part of the USBRL Project having length $11.2 \mathrm{~km}$ long, passes through the Pir Panjal Range of middle Himalayas in Jammu and Kashmir. The system will effectively calculate how much time will required to fill tunnel so travelers get the information about flooding.

\section{ACKNOWLEDGMENTS}

We would like to thank Dr. Tiwari H. U. for his contribution in offering guidance for this project. We also thank Dept. of Computer Engineering, PCCOER, Pune, India for valuable suggestions.

\section{CONCLUSION}

The application of urban flood control management system which is based on Internet of things technology provides comprehensive monitoring and pays more attention to the management of urban drainage and flooding prevention. It also provides operational services, conducts the monitoring and realizes the comprehensive supervision management, early warning management, and investigation management, which has not only improved the flood control decision making and drainage, but the city's flood control capacity is significantly improved. It will contribute to the security of society and harmonious development and construction.

\section{REFERENCES}

[1] Qinggong Ma, Bo Yang, Jipeng Wang, "Application of Internet of Things in Urban Flooding Prevention Management System", Scientific Research Publishing, Advances in Internet of Things, 2017, 7, 1-9, ISSN Print: 2161-6817.

[2] U.S. De, G. P. Singh and D. M. Rase, Urban flooding in recent decades in four mega cities of India, J. Ind Geophys. Union, April 2013,Vol.17, No.2, pp. 153-165.

[3] Xue, X.G., Jiang, S.F., Li, M., et al.(2016) Application Trend of GIS in Urban Drai-nage and Flooding Prevention. Science \& Technology Vision, No. 1, 71-72.

[4] Tong, L.G. (2015) Urban Drainage and Flooding Prevention Intelligent Controlling Platform Based on Internet of Things and GIS. Science \& Technology Information, 13, 10-12

[5] Asogwa, C.O., Zhang, X.M., Xiao, D.G., et al . (2011) Experimental Analysis of AODV, DSR and DSDV Protocols Based on Wireless Body Area Network. The 2011 International Workshop on Internet of Things Technology and Innovative Application Design, 597602

[6] Qian, Z.H. and Wang, Y.J. (2012) Study on Internet of Things and Its Application. Chinese Journal of Electronics , 40, 1023-1029.

[7] Gao, J., Zhu, Y.L., Li, L.F., et al . (2011) Routing Optimization Based on Ant Colony Algorithm for Wireless Sensor Networks with Long-Chain Structure. The 2011 International Workshop on Internet of Things ' Technology and Innovative Application Design, 434438 